\title{
Geometric Phase Circular Gratings Fabricated by Ultrafast Laser Nanostructuring for Symmetric Simultaneous Spatio-Temporal Focusing
}

\author{
Rokas Drevinskas, ${ }^{1}$ Aabid Patel, ${ }^{1}$ Ausra Cerkauskaite, ${ }^{1}$ Charles G. Durfee, ${ }^{2}$ and Peter G. Kazansky ${ }^{1}$ \\ 1. Optoelectronics Research Centre, University of Southampton, Southampton, SO17 1BJ, United Kingdom \\ 2. Department of Physics, Colorado School of Mines, Golden, Colorado 80401, USA
}

The recent advances in flat optics have challenged the limitations of conventional optics by implementing planar elements that instead of rely on dynamic phase manipulate light waves via subwavelength-spaced phase shifters with spatially varying phase response [1]. One of the approaches for designing geometric (Pancharatnam-Berry phase [2]) phase optical elements (GPOE) is to exploit the transparent dielectrics which originate form birefringence. The desired phase pattern of the wave is directly encoded in the optical axis orientation, and is equal to twice the rotation angle of the local retarder. A decade ago, the formation of selforganized subwavelength periodicity structures, referred to as nanogratings, in the bulk of silica glass after irradiation with ultrashort light pulses was observed [3]. Such a periodic assembly behaves as a uniaxial birefringent material with optical axis oriented parallel to the polarization of incident laser beam, and serves as a perfect candidate for designing geometric phase profiles of nearly any optical components. The performance of fabricated GPOE varies depending on the processing conditions reaching the efficiency and transmittance higher than $90 \%$, as well as the phase gradient higher than $\pi \mathrm{rad} / \mu \mathrm{m}$ [4]. The silica glass based optics with a damage threshold of $22.8 \mathrm{~J} / \mathrm{cm}^{2}$ demonstrate the potential of high-power applications.

Here we propose a direct-write ultrafast laser nanostructuring of silica glass as a method capable of fabricating circular gratings (CG) (Fig. 1(a)) for symmetric simultaneous spatio-temporal focusing (SSTF). The basic concept of the technique is to pre-chirp the laser pulse in space and time before the focusing optics [5]. The focusing lens then acts as a pulse compressor, producing the shortest pulse duration and highest intensity only at the focus of the beam. SSTF is typically implemented using grating-lens combinations or spatial light modulators but these setups are complicated to align and lead to unwanted spatio-temporal distortions.
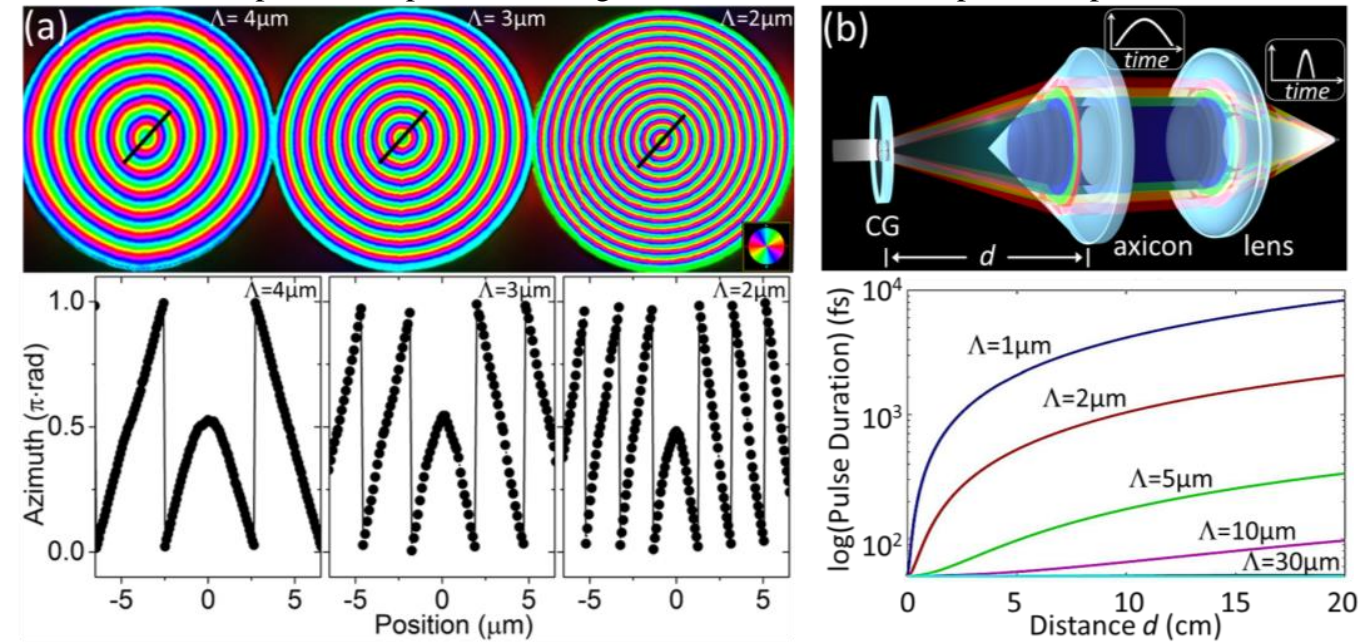

Fig. 1 (a) Circular grating converters (top) and its corresponding phase profiles (bottom) observed under polarization sensitive microscope. Left to right, periodicity of CG: $4 \mu \mathrm{m}, 3 \mu \mathrm{m}$ and $2 \mu \mathrm{m}$. Pseudo colors (inset) indicate the local azimuth of slow axis of laser induced nanogratings. (b) (top) Schematic of SSTF technique with observed symmetry, splitting the wavelengths in symmetric rings. (bottom) Simulated global pulse durations as a function of separation distance for varying grating periodicities at $\lambda=750 \mathrm{~nm}$ with $\Delta \lambda=30 \mathrm{~nm}$.

We demonstrate the use of a CG and axicon combination that maintains a symmetric SSTF with variable spatial-temporal control with the position of the axicon that can stretch the input beam in the region around the focus of a lens (Fig. 1(b)). In this case, the spatial chirp has cylindrical symmetry. This would eliminate directional dependence observed in laser writing [6,7]. The scheme improves beam profile at the focus removing pulse front tilt asymmetry. We envision that this development will be beneficial for the laser material processing community, biology and medicine where nonlinear microscopy is one of the major characterization tools.

\section{References}

[1] N. Yu and F. Capasso, "Flat optics with designer metasurfaces," Nat. Mater. 13, 139-150 (2014).

[2] Z. Bomzon, et al., "Pancharatnam-Berry phase in space-variant polarization-state manipulations with subwavelength gratings," Opt. Lett. 26, 1424-1426 (2001).

[3] Y. Shimotsuma, et al., "Self-organized nanogratings in glass irradiated by ultrashort light pulses," Phys. Rev. Lett. 91, 247405 (2003).

[4] R. Drevinskas, and P. G. Kazansky, "High-perfornance geometric phase elements in silica glass," submitted.

[5] G. Zhu, et al., "Simultaneous spatial and temporal focusing of femtosecond pulses," Opt. Express 13, 2153-9 (2005).

[6] P. G. Kazansky et al., "Quill” writing with ultrashort light pulses in transparent materials," Appl. Phys. Lett. 90, 151120 (2007).

[7] D. Vitek, et al.,"Spatio-temporally focused femtosecond laser pulses for nonreciprocal writing in optically transparent materials," Opt. Express 18, 24673-24678 (2010). 